\title{
Management of obstructed afferent-loop due to recurrence of pancreatic adenocarcinoma using an enteral self-expanding metal stent
}

\begin{abstract}
Background: Pancreatic cancer is frequently diagnosed in advanced stages. It is currently the fourth cause of cancer-related death among men and women. According to the American Cancer Society, prevalence in the US in 2017 corresponds to $3 \%$ of total malignant neoplasms. Afferent loop obstruction is an unusual severe complication following Whipple surgery. Its prevalence is $2.5 \%$ according to recent studies but it may be as high as $13 \%$, according to Pannala et al. in 2011. The most common clinical picture is acute cholangitis.

Case report: Eighty-year-old male with previous surgical intervention due to pancreatic head adenocarcinoma. After having tumor recurrence controlled by several chemotherapy courses, he presented with symptoms of acute cholangitis. On enteroscopy, a malignant stenosis in afferent loop was observed. It was subsequently treated with placement of a self-expanding enteral stent achieving complete resolution of symptoms and biochemistry.

Discussion: In the past, surgery was the only palliative therapeutic option available for afferent loop syndrome. Currently, similar palliative care has shifted towards less invasive techniques due to its lower morbi-mortality and complication rate. We present a case successfully treated with advanced therapeutic endoscopy. With the appearance of new and modern endoscopic equipment it is becoming possible to optimize treatment in a more heterogeneous group of patients, such as those with altered anatomy.
\end{abstract}

Conclusion: We present a case of malignant obstruction of afferent loop treated with advanced therapeutic endoscopy for palliative management with excellent clinical response and lower morbidity.

Keywords: pancreatic adenocarcinoma, malignant afferent loop syndrome, selfexpanding metallic enteral stent
Volume 8 Issue 2 - 2017

Josué J Aliaga Ramos,' Juan A Chirinos

'Médico residente de gastroenterología del $\mathrm{HCH}$, Peru

${ }^{2}$ Medico gastroenterólogo de la Clínica Anglo Americana, Peru

Correspondence: Josué Aliaga Ramos, Médico residente de gastroenterología del HCH, Calle Walter Stubbs N. 229. Lurigancho-Chosica, Lima- Peru, Tel 961-100-593, Email arjosue300@gmail.com

Received: August 25, 2017 | Published: December 06, 2017

\section{Introduction}

Pancreatic cancer is one of the most common gastrointestinal neoplasms. It has a cumulative incidence of 8.8 per 100,000 inhabitants. It is rare before the age of 45 but it abruptly becomes more common from this age on. ${ }^{1,2}$ Surgical resection is the only potentially curative treatment in patients with resectable disease, whereby the standard operation is a cephalic pancreaticoduodenectomy. However, its long-term success rate is low. ${ }^{3}$ Large series show that even among patients properly selected with curative resective surgery the 5-year survival rate is 10.5 to $25 \%$, presenting an average survival between 10.5 and 20 months. ${ }^{3,4}$

The afferent loop syndrome is a recognized complication following Whipple surgery. The main etiologies of late-stage afferent loop syndromes are tumor recurrences, adhesions, radiation-induced stenosis or internal hernias. ${ }^{5}$ The average appearance time of the latter is 1.2 years, whereas - according to a few current reports - the most frequent cause is a radiation-induced enteropathy (37.5\%) followed by tumor recurrences $(33 \%)$ and adhesion syndromes $(17 \%),{ }^{6,7}$ Most reports show that the main primary neoplasms generating a malignant afferent loop syndrome are gastric and pancreatic cancer following Billroth II surgery or Whipple procedure, respectively.

We present a case of an eighty-year-old male patient who presented with cholangitis symptoms secondary to an afferent loop obstruction due to tumor recurrence of pancreatic adenocarcinoma previously resected with classic Whipple procedure. It was managed with advanced therapeutic endoscopy techniques, with favorable outcome and complete symptomatic remission. This report would be the first of its kind in Peru and Latin America.

\section{Clinical case}

Eighty-year-old male patient with past medical history of poorly differentiated pancreatic head ductal adenocarcinoma with node affectation (pT3N1) in November 2013. He underwent classic cephalic pancreaticoduodenectomy (Whipple surgery), with positive CK7 immunohistochemistry. On follow up he received multiple chemotherapy and radiotherapy courses due to constant small recurrence at surgery site, achieving a good clinical response, assessed as no growth of recurrent tumor for 3years. Unfortunately, it was surgically unresectable due to its perivascular situation.

After 6months of his last chemotherapy, patient was referred to our institution with fever, jaundice and abdominal pain. On physical exam: Blood pressure 120/80 $\mathrm{mmHg}$; heart rate: 105 beats/minute, respiratory rate: 20 respirations/minute, and temperature: $38.0 \mathrm{OC}$. Patient was pale, with mild jaundice affecting mucous membranes. Abdominal exam showed mild pain on deep palpation of right upper quadrant. The rest of physical examination was unremarkable. He denied intake of any other drugs, herbs, tobacco and/or alcohol. Laboratory tests on admission: hemoglobin: $11.5 \mathrm{mg} / \mathrm{dL}$, hematocrit: 36\%, MCV: 88.1, MCH: 27.9, MCHC: 31.7, leukocytes: 7,500/mm3, platelet count: $357,000 / \mathrm{mm} 3$. Liver test showed alkaline phosphatase: $432 \mathrm{mg} / \mathrm{dL}$, GGT: $500.8 \mathrm{mg} / \mathrm{dL}$, total bilirubin: $1.59 \mathrm{mg} / \mathrm{dL}$ (DB: 0.81 , 
IB: 0.78), total proteins: $6.39 \mathrm{mg} / \mathrm{dL}$ (albumin: 3.80, globulins: 2.59 ), GOT: 63 and GPT: 128.

CT scan revealed dilation of both the bile duct and afferent loop. An upper endoscopy was programmed with possible frontal view endoscopic retrograde cholangiopancreatography. During the procedure, at the level of the distal antrum, two anastomosed intestinal loops were found: one of them occupied $90 \%$ of lumen and had dry content and the other limb had bile inside. We progressed under fluoroscopy through the latter identifying an eccentric, irregular stenosis with nodular-looking tissue, described as "pearls," with abnormal vasculature at approximately $25 \mathrm{~cm}$ (Figure 1). Several biopsies were taken. Fluoroscopy showed a 2-cm-long stenosis surrounded by pronounced U-shaped angulation.

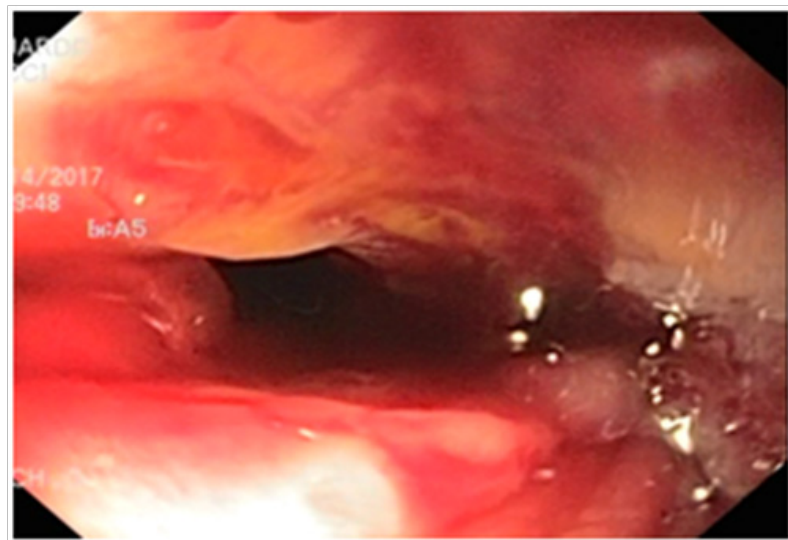

Figure I Afferent loop stenosis.

The enteric stenosis of the afferent loop was dilated using an $8-10 \mathrm{~mm}$ balloon without being able to pass due to endoscopic loop seen on fluoroscopy. The therapeutic frontal endoscope was then replaced by a single balloon enteroscope, being able to reduce the loop with the overtube. After passing through the stenosis with moderate resistance, the bile duct was identified and found to be dilated but without any abnormalities at the level of biliary-enteric anastomosis. A direct choledochoscopy was performed, revealing absence of obstructive disease at that level (Figures $2 \mathrm{a} \& 2 \mathrm{~b}$ ). After the procedure, the patient presented partial improvement, both clinically and biochemically. Biopsies revealed a well-differentiated tubular adenocarcinoma with the same histological and immunohistochemically characteristics as the primary disease at pancreatic level. Sixdays later antegrade balloonassisted enteroscopy was performed and an endoscopic endoprothesis was placed for further palliative management. After passing through the stenosis and performing cholangiography, the proximal and distal border of the stenosis were delineated under fluoroscopy using metallic marks. Next, a 0.035 -inch guide was placed (Figure $3)$. Afterwards, the enteroscope was replaced with a therapeutic endoscope in order to be able to place a $22 \mathrm{x} 120 \mathrm{~mm}$ self-expanding metallic stent through the endoscopic working channel, model Niti-S D type stent (Tae-Wong medical) (Figures $4 \& 5$ ). Before ending the procedure, complete opening of both ends and central waist at stenotic level was observed. Furthermore, proper flow of contrast dye through afferent loop was verified at the end of the procedure (Figure 6). 6 months later, and until this paper was finished, the patient continues to be asymptomatic, with full biochemical resolution. Control image study shows correct placement of endoscopic endoprothesis (Figures $7 \mathrm{a} \& 7 \mathrm{~b}$ ), as well as bile duct and afferent loop with diameters within normal ranges.
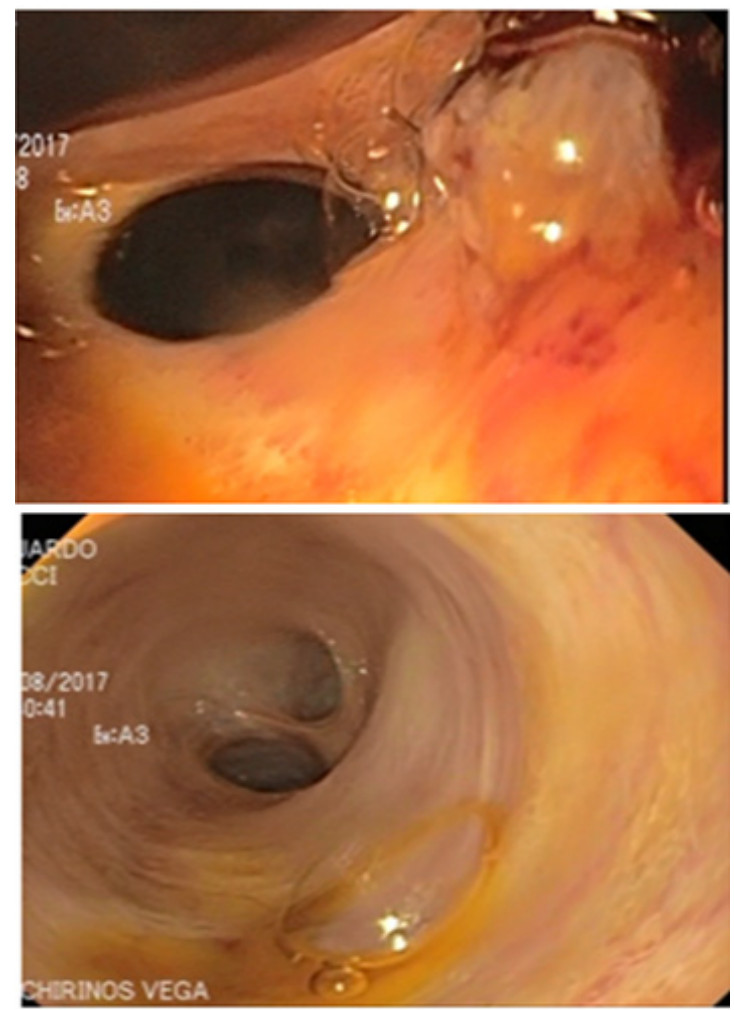

Figure 2a 2b Panoramic view of biliary-enteric anastomosis.

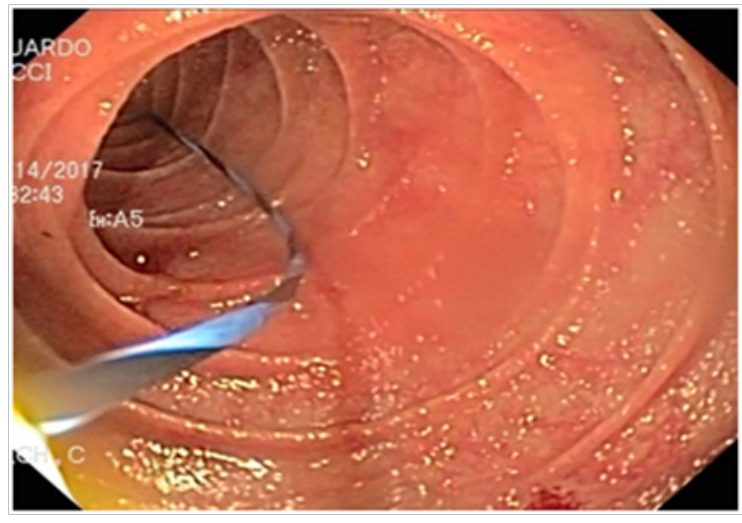

Figure 3 Placement of guidewire at stenosic site.

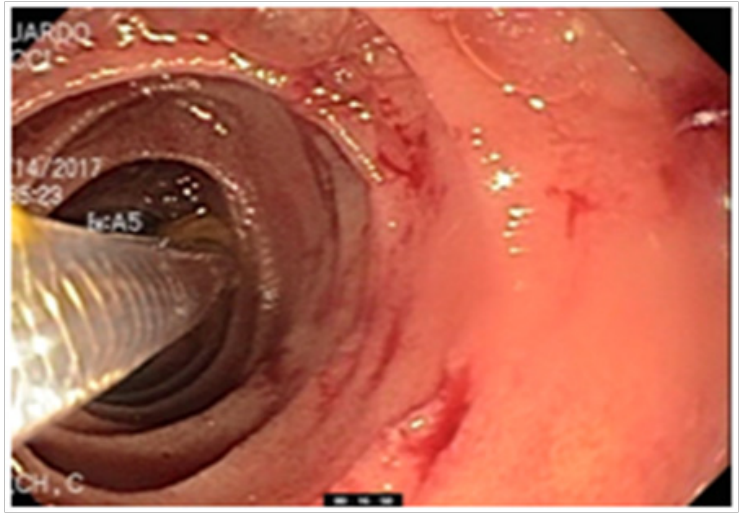

Figure 4 Insertion of enteral endoprosthesis. 


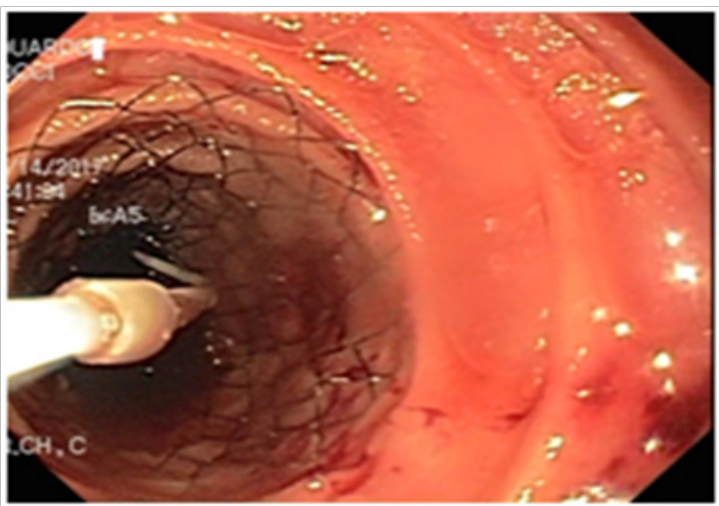

Figure 5 Deliver of enteral endoprosthesis.

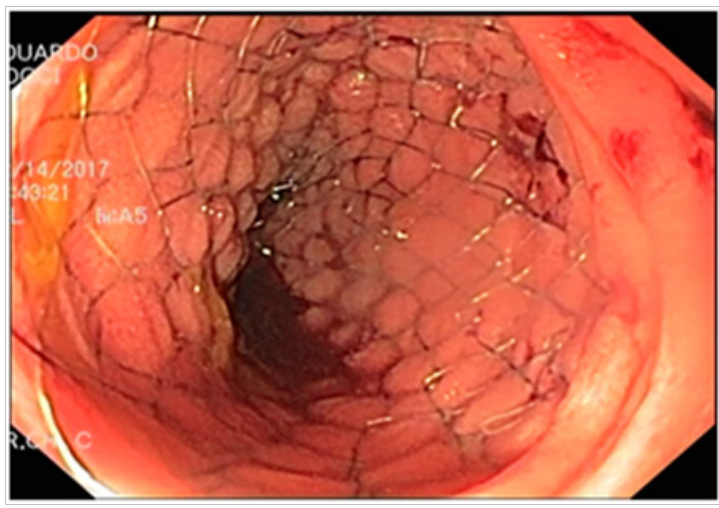

Figure 6 Successfully placed self-expanding stent.
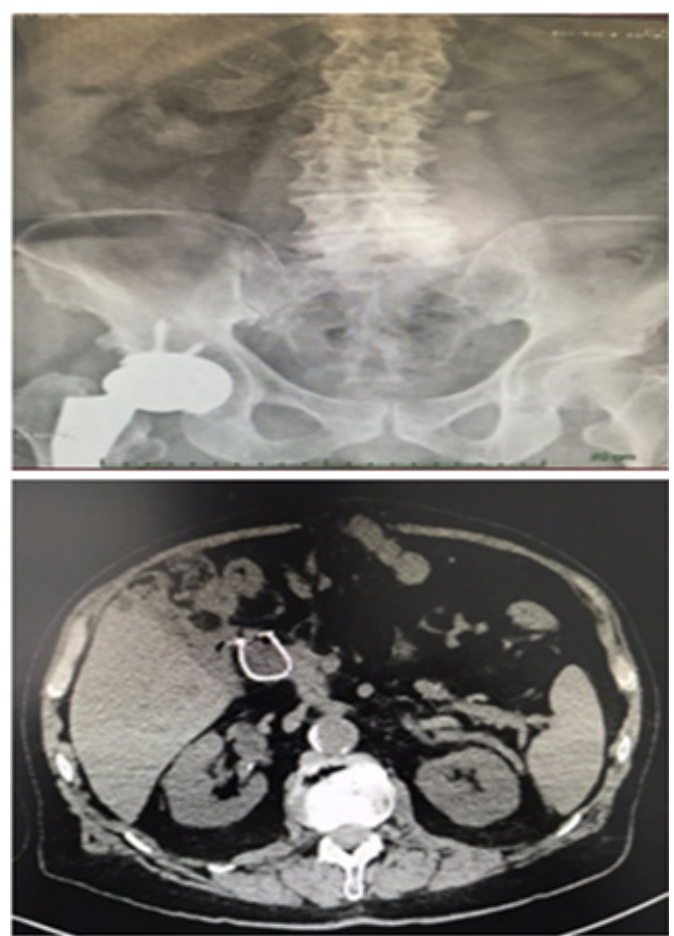

Figure 7a, 7b Post-endoprosthesis image control with non-dilated biliaryenteric anatomy.

\section{Discussion}

The afferent loop syndrome is a relatively frequent complication, as high as $13 \%$ according to Pannala et al., ${ }^{1-3}$ The etiology of this syndrome can be quite varied. The main cause of this complication among post-surgery patients following a Whipple procedure is tumor recurrence, more so if patients were subjected to intensive chemotherapy protocols, ${ }^{4,5}$ similar to our case. According to clinical and radiographic characteristics, the afferent loop syndrome can be sub-classified into acute and chronic. The first category appears during the early postoperative period, while the second one appears a few years later, ${ }^{6,7}$ as was the case for our patient.

The first reported case of afferent loop syndrome was described by Mc Nealy in 1942 as a consequence of postoperative anastomotic duodenal stump leakage. There are few studies regarding the diagnosis and treatment of this clinical entity the majority being case reports. Ruiz Pardo et al. ${ }^{8}$ presented a case report of a 54-year-old woman who underwent a cephalic pancreaticoduodenectomy due to pancreatic head adenocarcinoma who - after intensive chemotherapy, presented tumor recurrence at afferent loop level, appearing as cholangitis, which was surgically resolved without any complications. The case we present is very similar to Ruiz Pardo's report, ${ }^{8}$ however, our patient was managed with advanced therapeutic endoscopy techniques, with results equally effective to those previously described.

Worldwide, there are few case reports that use an endoscopic approach to treat this clinical entity. Matsumoto ${ }^{9}$ in Japan treated an eighty-year-old patient with malignant afferent loop syndrome caused by duodenal carcinoma performing transgastric drainage via endoscopic ultrasonography. In this case the bile duct was not dilated and affected afferent loop was located close to gastric cavity. It was combined with placement of self-expanding metallic stent in the anastomosis, generating positive short- and mid-term results. This differs from the patient we present with regard to the type of endoscopic approach used, because in our case there was a dilated bile duct with cholangitis and the affected afferent loop was not close to the stomach. However, this report strengthens the use of self-expanding metallic stents in palliative treatment of malignant neoplasms of the gastrointestinal tract. ${ }^{10,11}$

In the past, the only feasible treatment for correcting a malignant afferent loop was surgically. However due to great progress in therapeutic digestive endoscopy, it has been possible to solve these problems with a lower morbi-mortality and complication rate. One of the main endoscopic techniques used in these cases is the placement of self-expanding metallic stents for bile drainage, with high success rates when conducted by experienced hands, and preventing external percutaneous access through interventional radiology, which could give rise to mid- and long-term complications. As described by A Sakai et al. ${ }^{12}$ in their report of a 66-year-old male patient with malignant afferent loop syndrome due to recurrence of a pancreatic cancer tumor, where a self-expanding metallic stent with a diameter of $22 \mathrm{~mm}$ and a length of $8 \mathrm{~cm}$ was used on the stenosis site, after decompressing the dilated afferent loop with a nasojejunal tube of 7.5 Fr for sixdays, with optimum short- and mid-term results. In our patient, we also used a metallic endoprosthesis similar to the case reported, but through a 0.035 -inch guide placed with initial help of a balloon-assisted enteroscope, due to the particular narrowness of the lesion, followed by therapeutic endoscope to deliver the stent.

New endoscopic techniques are being used with modern equipment that has recently been developed with the purpose of optimizing benefits, reducing complications and improving the patients' quality 
of life. ${ }^{13}$ One of the first studies on this subject was Shugo et al. ${ }^{14}$ case report, in which a balloon-assisted enteroscope was used to correct a malignant afferent loop syndrome caused by recurrence of pancreatic head cancer in a 76-year-old male patient. On this patient, a balloonassisted enteroscope was used, with a guide providing help for placing a metallic endoprosthesis, due to the inability to gain access with conventional methods. It was possible to identify a 4-cm-long stenosis $30 \mathrm{~cm}$ from the anastomosis, as well as to place an uncovered pyloric/ duodenal Niti-S self-expanding enteral metallic stent of $22 \times 100 \mathrm{~mm}$ through a guide, with successful results, resolving jaundice a few days after the procedure.

However, one of the main and most recent research studies based on this ground-breaking endoscopic progress was the one published at the beginning of 2017 by K Tsutsumi et al., ${ }^{15}$ also in Japan, which highlights the great qualities and benefits of using a short doubleballoon enteroscope to treat patients with surgically altered anatomy. Three patients were analyzed, one of them with recurrent benign biliary obstruction - a young female patient with bile duct atresia who had a hepaticojejunostomy with liver transplant, and two patients post-gastrectomized due to advanced gastric cancer, one of whom developed a malignant afferent loop syndrome and the other one a biliary obstruction, also due to tumor recurrence. In the 3 patients short double-balloon enteroscopy was used, with a working channel widened to $3.2 \mathrm{~mm}$, thanks to which it became faster, safer and more effective to place the endoprosthesis at different levels. Moreover, it was pointed out that the use of the double-guide technique is quite helpful when placing multiple stents. So, this novel endoscopic technique is presented as a first-line, in both diagnosis and treatment of patients with surgically altered anatomy.

\section{Conclusion}

In conclusion, the afferent loop syndrome is a clinical entity that is rather infrequent in daily clinical practice. However, early recognition helps timely therapeutic treatment to improve patients 'quality of life. New progress in gastrointestinal therapeutic endoscopy with new equipment and more flexible and safer stents allows good palliative with potentially lower morbidity and fewer complication rates. More studies should be carried to determine the full role of these promising techniques in patients with surgically altered anatomy.

\section{Conflicts of interest}

There is no conflict of interests in the study we present.

\section{Acknowledgements}

None.

\section{Funding}

None.

\section{References}

1. Sleisenger M, Friedman L, Feldman M. Enfermedades Gastrointestinales y Hepáticas. ( $8^{\text {th }}$ edn), Tomo II. Cap. 2004;84. pp.1762-1763.
2. Statistics for pancreatic cancer. American Cancer Society's. United States. 2007.

3. Pannala R, Brandabur J, Gan S, et al. Afferent limb syndrome and delayed gastrointestinal problems after pancreaticoduodenectomy for pancreatic cancer: single-center, 14-year experience. Gastrointest Endosc. 2011;74(2):295-302.

4. Hosono S, Ohtani H, Arimoto $\mathrm{Y}$, et al. Endoscopic stenting versus surgical gastroenterostomy for palliation of malignant gastroduodenal obstruction: a meta-analysis. J Gastroenterol. 2007;42(4):283-290.

5. John Spiliotis, Demetrios Karnabatidis, Archodoula Vaxevanidou, et al. Acute cholangitis due to afferent loop syndrome after a Whipple procedure: a case report. Cases Journal. 2009;2:6339.

6. Kwong WT, Fehmi SM, Lowy AM, et al. Enteral stenting for gastric outlet obstruction and afferent limb syndrome following pancreaticoduodenectomy. Ann Gastroenterol. 2014;27(4):413-417.

7. Huang J, Hao S, Yang F, et al. Endoscopic metal enteral stent placement for malignant afferent loop syndrome after pancreaticoduodenectomy: Case report and review of literatura. Wideochir Inne Tech Maloinwazyjne. 2015; 10(2):257-265.

8. Jose Ruiz Pardo, Erik Llacer-Milla, Pilar Jimeno Griño, et al. Colangitis por obstrucción de asa aferente tras duodenopancreatectomia cefálica. Cirugía española. 2016;94(2):106-118.

9. Kazuyuqui Matsumoto, Hironari Kato, Takeshi Tomoda, et al. A case of acute afferent loop síndrome treated by transgastric drainage with EUS. Journal Gastrointestinal Endoscopy. 2013;77(1):132.

10. Bakes D, Cain C, King M, et al. Management of afferent loop obstruction from recurrent metastatic pancreatic cancer using a venting gastrojejunostomy: A case Report. World J Gastrointest Onco. 2013;1 5(12):235-239.

11. Han K, Song HY, Kim JH, et al. Afferent Loop Syndrome: Treatment by Means of the Placement of Dual Stents. AJR Am J Roentgenol. 2012;199(6):W761-W766.

12. Arata Sakai, Hideyuki Shiomi, Yoshihiro Okabe, , et al. Effectiveness of endoscopic self-expandable metal stent placement for afferent loop obstruction caused by pancreatic cancer recurrence after pancreaticoduodenectomy: A Case Report. Clin J Gastroenterol. 2015;2(3):122-125.

13. Shimatani M, Takaoka M, Tokuhara M, et al. Through-the-scope selfexpanding metal stent placement using newly developed short doublé balloon endoscope for the effective management of malignant afferentloop obstruction. Endoscopy. 2016;48:E6-E7.

14. Haruhiko Shugo, Yuji Hodo, Manabu Yoneshima. Endoscopic Metallic Stent Insertion for Malignant Afferent Loop Obstruction Using BalloonAssisted Enteroscopy: A Case Report. The American Journal of Gastroenterology. 2015;110:356-357.

15. Tsutsumi K, Kato H, Okada H. Impact of a Newly Developed Short Double-Balloon Enteroscope on Stent Placement in Patients with Surgically Altered Anatomies. Gut Liver. 2017;11(2):306-311. 